\title{
Indicators of the effectiveness of self-healing asphalt concrete
}

\author{
Sergey Inozemtcev ${ }^{*}$ and Evgeniy Korolev \\ Moscow State University of Civil Engineering, Yaroslavskoe Shosse, 26, Moscow, 129337, Russia
}

\begin{abstract}
In the article, indicators reflecting the degree of efficiency of the self-healing technology being developed are proposed the degree of restoration of the operational state of the structure (degree of self-healing); the speed of the process of restoring the operational state of the structure (self-healing rate); durability operational status after self-healing; timeliness of initiation of the self-healing process. Indicators of the effectiveness of self-healing technology are justified by using the Pavement Condition Index (PCI).
\end{abstract}

\section{Introduction}

The search for scientifically based technology solutions to reduce maintenance costs and increase the service life of pavement is the target of scientific research about asphalt concrete [1...5]. It is known that increasing the strength of asphalt does not lead to an increase in its resistance to various operational and climatic factors [6...8]. Regularly increase asphaltic concrete strength at positive temperatures increases the strength at low temperatures [9...14]. This fact is described in the framework of classical concepts of composite materials (structural model), in which at the interface of the phase "binder dispersed phase" a layer of binder is formed with enhanced physical properties (film phase):

$$
R=\sigma_{s}\left(1-v_{p}\right)-\sigma_{s} v_{f}\left\langle\left(1-\rho_{f} S_{a} h_{0}\left(k_{m}-1\right)\right)\right\rangle+\sigma_{f}^{\prime} v_{f}
$$

where $\sigma_{s}$ - coefficient characterizing the strength of free bitumen; $\sigma_{f}^{\prime}$ - coefficient characterizing the influence of the mineral part; $v_{f}$ - volume fraction of the mineral part; $v_{p}$ - pore volume fraction; $\rho_{f}$ - average density of the mineral part; $S_{a}$ - specific surface area of the mineral part; $k_{m}$ - binder hardening coefficient in the adsorption state; $h_{0}-$ optimum thickness of the binder.

A decrease in the strength of a composite is observed with a decrease in the thickness of the binder less than the optimal value of $h_{0}$. In this case, a decrease in the strength of the composite occurs with an increase in its porosity due to the lack of binder and the formation of aggregates, which consist of particles of the dispersed phase [15]:

$$
R=\sigma_{s}\left[1-v_{p}-v_{f}^{\prime}\left\langle\left(1-\frac{1-v_{f, o p t}}{v_{f, o p t}}\left(k_{m}-1\right)\right)\right\rangle\right]+\sigma_{f}^{\prime} v_{f}^{\prime}
$$

\footnotetext{
* Corresponding author: inozemtsevss@mail.ru
} 
where $v_{f}^{\prime}$ - coefficient characterizing the influence of the mineral part; $v_{f, o p t}-$ optimal proportion of the mineral part(corresponds to the maximum strength).

The adequacy of this model is confirmed in [16]. The value $k_{m}$ is the control parameters of the model of composite, which characterizes the excess strength of the film phase of the binder, and the optimal thickness of the binder layer $h_{0}$. In [17] presents a method for determining the thickness of the adsorption layer of bitumen according to the results of rheological tests.

From (1) and (2), as well as from the inequality $h_{0}<2 h_{a}$ (here $h_{a}$ is the thickness of the adsorbed bitumen layer), it follows that depending on the physicochemical properties of the dispersed phase, asphalt concrete

* Corresponding author: inozemtsevss@ mail.ru

forms a balance between the adsorption and free bitumen. The establishment of such a balance requires additional studies of the structure of the layer of bitumen located between the particles of the dispersed phase. The minimum amount of binder is necessary for the enveloping of the grains of the mineral part and the formation of the boundary layer:

$$
V_{\min }=\sum_{\mathrm{i}=1}^{n}\left(\frac{d_{i}}{n \sqrt{R_{e}}} \cdot \pi d_{i}^{2}\right),
$$

where $R_{e}$ - Reynolds number; $d_{i}$ - the grain diameter of the mineral part of the $i$-th fraction.

The balance between the content of free and adsorbed bitumen should also affect the performance properties. Usually, the substance in the adsorption state is characterized by other indicators of properties (density increases, phase transitions of the first kind decrease, etc.), the packing density of molecules increases and their mobility is limited, which is equivalent to a decrease in the "structural temperature" of the substance. It is assumed that bitumen in the adsorbed state will better resist the action of physical and chemical factors compared to bitumen in a bulk (free) state.

For asphalt, this means that a certain ratio between strength at positive temperatures and crack resistance should be established for ensuring durability. Durability represents the maximum possible lifetime of the asphalt concrete pavement, during which its operational suitability is maintained and it is not repaired. At the same time, irreversible processes occur in asphalt concrete, leading to the formation of defects and the disruption of the integrity of the structure due to weather and climate impacts and the load from vehicles.

The durability of asphalt concrete is inversely proportional to the speed of flow of destructive processes and their intensity. Thus, in order to ensure an increase in the durability of the pavement, it is necessary to develop a method for maintaining the state of the structure of asphalt concrete without defects. This is possible by giving the asphalt concrete unique properties: self-repair the integrity of the composite and its ability to resist external factors.

Application of the encapsulated modifier would ensure restoration of the integrity of the composite and its ability to resist external influences during the formation of defects [18].

Increasing the durability of the sidewalk can be achieved using self-healing technology, which is implemented using an encapsulated modifier. Capsules containing the modifier are added to the asphalt mix at the final stage of preparation and during the initial structure formation act as inert filler. The temperature of the preparation of the asphalt mix should not have a destructive effect on the capsule shell and the encapsulated modifier. The shell 
material of the capsules must ensure their integrity in the compacting the asphalt concrete mixture, preventing premature release of the modifier.

During the operation of the pavement, there is a violation of the integrity of the structure of asphalt concrete under the influence of operational factors (the formation of cracks and other defects). The mechanism of release of the modifier from the capsules is initiated by defect formation (discontinuity of the structure), which destroys the capsules. After that the modifier flows out through the formed defect in the shell, partially fills the crack space, wetting the walls, and diffuses into the bitumen.

Then the stage of secondary local structure formation begins: the modifier polymerizes and the integrity of the composite is restored, the ability to resist external influences. Polymerization of the modifier is carried out in its interaction with the hardener, which was deposited on the outer surface of the capsules and/or is present in the volume of bitumen in the form of an inert additive. Thus, the polymerized site of the binder ensures that it corks cracks and stops its development. Also, local areas of the elastic polymer-bitumen binder are able to more dissipate the dynamic load from vehicles.

The considered example of self-healing technology is the most promising in the direction of road construction. The formulation of the scientific and practical bases of selfhealing technology will make it possible to extend the life of a pavement when it is used.

\section{Materials and Methods}

The main criteria for the effectiveness of self-repairing asphalt concrete were described using a model for estimating and predicting the operational state of the pavement based on the calculation of the index of the operational state (Pavement Condition Index (PCI)). The calculation of the index is based on the following provisions [19]:

- the indicator should reflect the cyclical nature of climatic and mechanical impacts;

- the indicator should take into account the heterogeneity of the safety margin for cyclic climatic and mechanical effects;

- the number of cycles of climatic and mechanical factors is assumed to be proportional to the time of pavement operation.

Evaluation of the operational state of the pavement in terms of PCI was carried out on a scale from 0 ("Failed") to 100 ("Good") and was calculated by the formula [20]:

$$
q(t)=P C I(t)=100 \cdot \Phi\left(\frac{1}{c}\left(1-\left(\frac{t}{T}\right)^{\frac{1}{b}}\right)\right),
$$

where $\Phi(x)$ - probability integral, for which there are detailed tables and approximate formulas;

$t$ - service life, year;

$T$ - the period for which the quality will be halved, a year;

$b=2.5 \ldots 3.5$ - coefficient characterizing the resistance of the material to dynamic loads; $c=0.16 \ldots 0.20-$ coefficient of variation, reflecting the heterogeneity of the safety margin.

\section{Results and discussion}

The quality of self-healing asphalt concrete is determined by the parameters of the initial structure of the material and indicators reflecting its state in the initial period of operation (figure 1). 


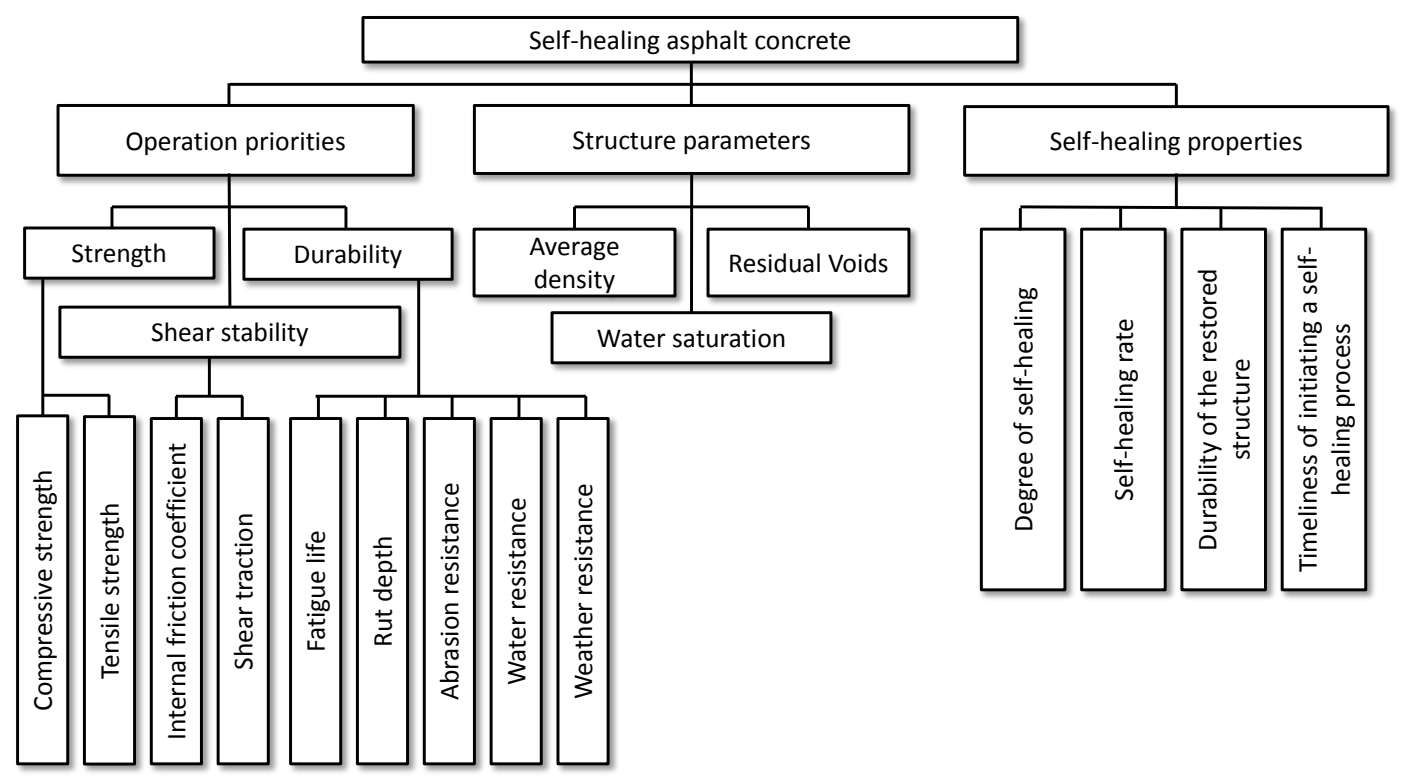

Fig. 1. Quality indicators of self-healing asphalt concrete.

The regulatory documents (GOST 12801-98) reflect the indicators of the structure (average density, water saturation, residual voids) and indicators of the state of the structure (strength and shear stability). The criteria system also includes indicators reflecting the ability of asphalt concrete to resist cyclic mechanical loads and the effects of weather factors [21].

The effectiveness of self-healing technology is determined by the ability to independently restore the state of the structure or improve the operational state. The practical significance of using self-healing technology is the restoration of the ability of asphalt concrete to resist external factors without additional energy and financial costs.

Indicators to assess the effectiveness of self-healing technology are:

- the degree of restoration of the operational state of the structure (degree of selfhealing);

- the speed of the process of restoring the operational state of the structure (selfhealing rate);

- durability operational status after self-healing;

- timeliness of initiation of the self-healing process.

The main criterion for the effectiveness of self-healing technology is the degree of restoration of the operational state of the pavement $(\Delta q)$, which is ensured by the process of secondary structure formation using an encapsulated modifier when the equality $q_{1}+\Delta q=$ $q_{0}$ (fig. 2) is fulfilled. 


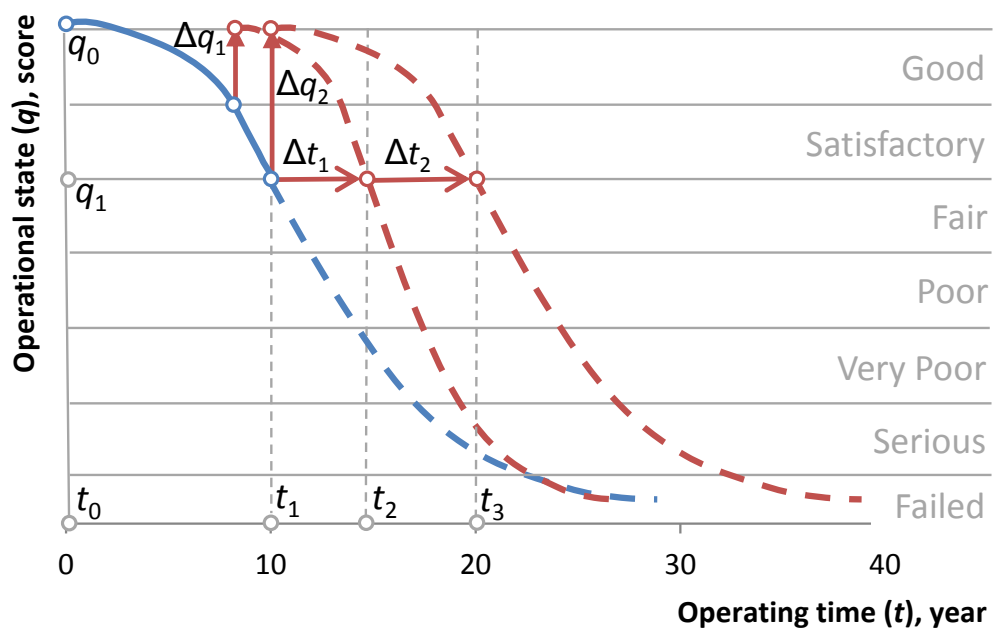

Fig. 2. The influence of the efficiency and timeliness of the recovery to the operational state of the pavement.

The maximum efficiency of restoring the operational state of the pavement is achieved when this process is initiated in a timely manner and when the condition $\Delta q=q_{0}-q_{1}$ is fulfilled. The process of restoring the operational state should be initiated when the condition of the coating allows safe and comfortable operation, because when the pavement condition is "Fair", road services begin repairing the coating.

Since the restoration of the operational state of the pavement occurs as a result of the interaction of the modifier from the capsule with the external environment, the effectiveness of self-healing will be inversely proportional to the speed of this process (fig. $3)$.

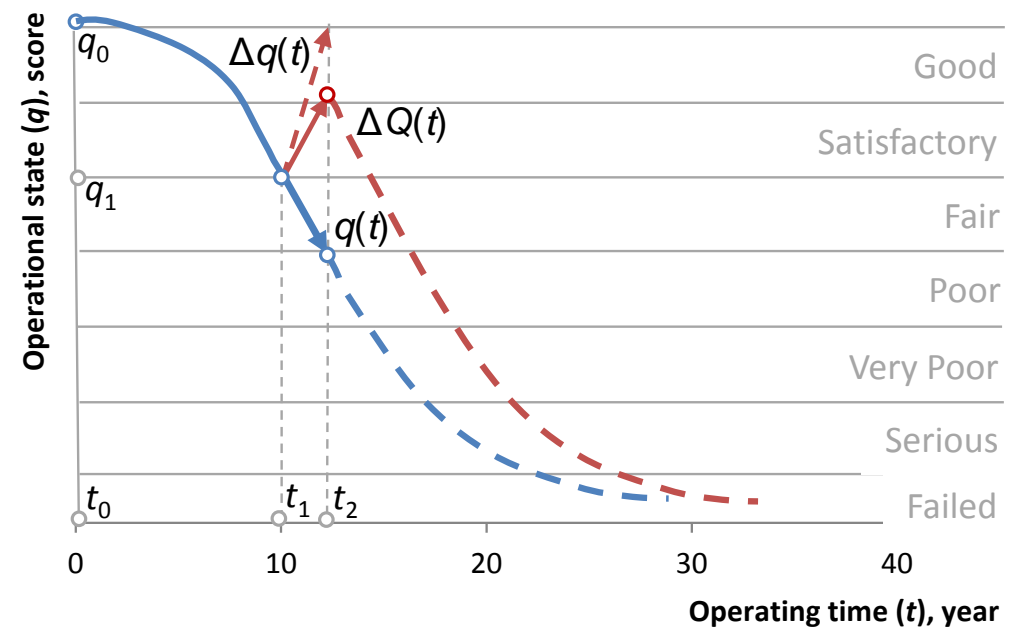

Fig. 3. The influence of the speed of the recovery to the operational state of the pavement.

Thus, in the case of the duration of the self-healing process of asphalt concrete, the dependence of the change in operating state of the pavement on time will be presented as a function of $\Delta q(t)$. However, when developing a self-healing technology, it is necessary to 
take into account that during recovery, destructive processes $q(t)$ affect the operational state of the pavement, which reduces the final efficiency at the end of the process. In this case, the change in the operation state of the pavement will be determined by the sum of multidirectional processes $\Delta Q(t)=\Delta q(t)-q(t)$. That is, ensuring the effectiveness of selfhealing technology is possible when the recovery rate of the operational state of the pavement is greater than or equal to the speed of destructive processes.

The process of restoring the operational state of the material is completed when a new structure of asphalt concrete is formed, and the quality of the pavement will determine the effectiveness of self-healing technology (fig 4).

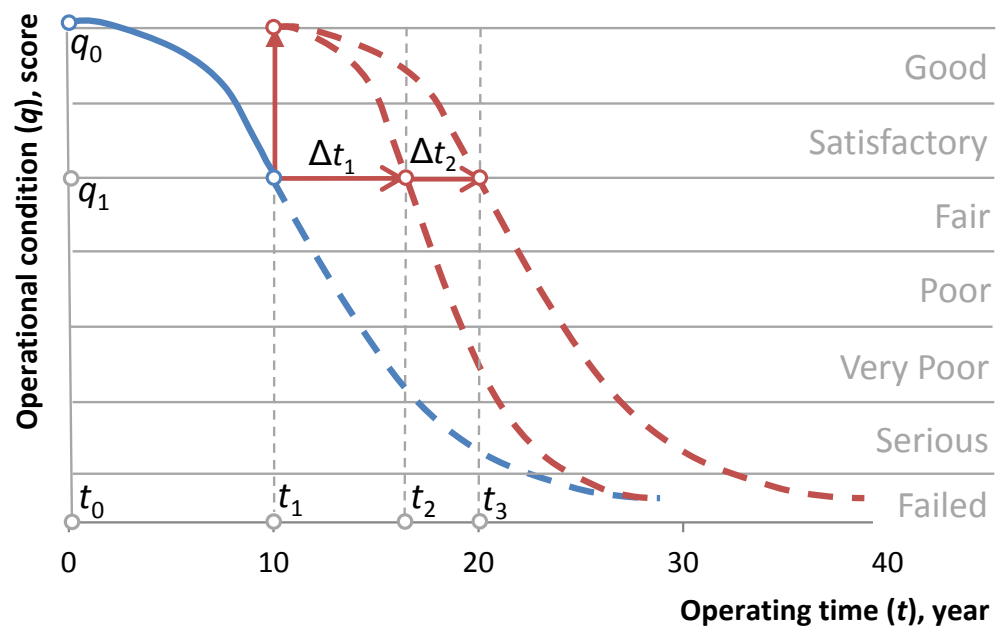

Fig. 4. The influence of the durability of the structure after the self-healing process to the operational state of the pavement.

Obviously, an increase in the maintenance-free life of the pavement is directly proportional to the quality of the combined asphalt concrete structure formed as a result of the self-healing process and its ability to resist external influences.

\section{Conclusion}

The effectiveness of self-healing technology is determined by the ability to independently restore the state of the structure or improve the operational state. Indicators that reflect the effectiveness of the self-healing technology being developed are the degree of restoration of the operational state of the structure (degree of self-healing); the speed of the process of restoring the operational state of the structure (self-healing rate); durability operational status after self-healing; timeliness of initiation of the self-healing process.

\section{References}

1. ShuangCui, Bamber R K, Blackman, Anthony J, Kinloch, Ambrose C and Taylor 2014 Int. J. of Adhesion and Adhesives 54 100-11.

2. Kishchynskyi S, Nagaychuk V and Bezuglyi A 2016 Proc. Eng. 143 119-27.

3. Van Oort W P 1956 Ind. Eng. Chem. 48 (7) 1196-201

4. Oliver John W H, 1992 Fuel Sc. and Tech. Int. 10 (4-6) 501-18.

5. Shuanfa Chen, Bowen Guan, Rui Xiong, Yanping Sheng and Rui He 2011 J. of Wuhan Un. of Techn.-Mater. Sci. Ed. 26 (6) 1200-04.

6. Kotlyarskiy E V and Voyeyko O A 2007 The durability of road asphalt pavements and 
factors contributing to the destruction of the structure of asphalt concrete during operation (Moscow: Moscow Automobile and Road Institute) p 136.

7. Kolbanovskaya A S and Davydova A R 1966 Structuring road bitumen Physicochemical mechanics of dispersed structures (Moscow: Science) pp 103-13.

8. Pechenyy B G 1981 Durability of bitumen and bitumen mineral coatings (Moscow: Stroyizdat) p 123.

9. Huachun Zhai and Delmar Salomon 2005 Trans. Res. Rec. J. of the Trans. Res. Board 1901(1) 44-51.

10. Higuera Sandoval C H , Camargo Amaya XV and Suárez Molano E A 2015 Ing. Unv. $192335-49$.

11. Feipeng Xiao, Punith V S, Serji N Amirkhanian and Bradley J Putmanb 2013 Can. J. of Civ. Eng. 40(9) 861-68.

12. Yongchun Cheng, Wensheng Wang, Guojin Tan and Chenglin Shi 2018 Sustainability 10, 2179.

13. Ruixia Li, Pravat Karki, Peiwen Hao and Amit Bhasin 2015 Cons. and Build. Mat. 96 47-54.

14. Inozemtsev S S and Korolev E V 2013 Vestnik MGSU 10 131-39.

15. Korolev E V, Bazhenov Yu M and Albakasov A I 2010 Radiation-protective and chemically resistant sulfur building materials (Penza, Orenburg: IPK OGU) p 364.

16. Korolev E V, Smirnov V A, Albakasov A I and Inozemtsev A S 2011 Nanotech.nologies in constr. 3 (6) 32-43.

17. Korolev E V, Inozemtcev S S and Smirnov V A 2016 Nanomodified bitumen composites: Solvation shells and rheology Adv. Mat., Str. and Mech. Eng. - Proc. of the Int. Conf. on Adv. Mat., Str. and Mech. Eng. 393-98.

18. Kryzhanovsky V K, Burlov V V, Panimatchenko A D and Kryzhanovskaya Yu V 2003 Technical properties of polymeric materials (S.-Petersburg) p 240.

19. Siti Nurjanah Ahmad, Tri Harianto, Lawalenna Samang and Muralia Hustim 2018 Level vulnerability damage of pavement using Pavement Condition Index method. MATEC Web of Conf. Vol. 181, 11003.

20. Radovskiy B S 2006 Road mach. 6 108-18.

21. Inozemtsev S S and Korolev E V 2019 Mat. Sc. Forum 945 147-157. 